Research Article

\title{
Experimental Investigation and Development of Multi Response ANN Modeling in Turning Al-SiCp MMC using Polycrystalline Diamond Tool
}

\author{
Santosh Tamang ${ }^{\dot{A}}$ and M.Chandrasekaran ${ }^{\dot{A}^{*}}$ \\ ${ }^{\dot{A}}$ Department of Mechanical Engineering, North Eastern Regional Institute of Science and Technology (NERIST), Nirjuli, Arunachal pradesh. INDIA
}

Accepted 10 January 2014, Available online 01 February 2014, Special Issue-2, (February 2014)

\begin{abstract}
Metal matrix composites (MMC) are widely used for producing components in automotive, aerospace and bio-medical industries because of their improved properties in comparison with non reinforced alloys. These materials are known as difficult-to-machine materials because of hard abrasive particles were used for reinforcement. Among these, the aluminium based silicon carbide particulate (Al-SiCp) reinforced MMC have received more commercial attention due to their high performance. In this work, machinability study and predictive model development was carried out for machining Al-SiCp MMC using polycrystalline diamond (PCD) tool. Turning experiments based on full factorial design $\left(3^{3}\right)$, a total of 27 machining trials are carried out to study the effect of turning parameters viz., spindle speed $(N)$, feed $(f)$ and depth of cut $(d)$ on the responses such as surface roughness $(R a)$ as product quality and material removal rate (MRR) as productivity improvement in the machining process. Multi response predictive modeling has been developed using artificial neural network (ANN). The ANN architecture having 3-6-2 is found to be optimum average percentage error of $4.46 \%$ for surface roughness and $7.26 \%$ for material removal rate. The predictive model exhibit close correlation with the experimental result as confirmed by the validation test. The methodology found to be effective tool and can be developed with minimum effort.
\end{abstract}

Keywords: $M M C$, Turning, ANN, Ra, MRR.

\section{Introduction}

Machining is one of the most widely used manufacturing processes. The process is highly complex and uncertainty in nature. Modeling and optimization of the machining process is very important for metal cutting industries to survive in the competitive world market by producing quality products at competitive price. Among various work materials available the introduction of composite material becomes popular recently and these are named as 'material of the future'. Composite materials are combination of two or more materials (i.e., reinforcing elements, fillers and binders) differing in form or composition on a macro scale. These are found application in industries such as automotive, aerospace, electronics, and military due to their attractive properties as high strength, low weight, high modules, low ductility, high wear resistance, high thermal conductivity and low thermal expansion by (Allison et al, 1993). The demerits of MMC include high cost of manufacturing, high machining cost and lack of knowledge in designing composites.

Among different machining processes turning is one of the commonly used process for the production of cylindrical surface using single point cutting tool.

*Corresponding author Santosh Tamang is a Research Scholar; M.Chandrasekaran is working as Associate Professor

DOI: http://dx.doi.org/10.14741/ijcet/spl.2.2014.01
Researchers have investigated on turning different composites viz., Poly ether ether ketone (PEEK), Composite fibre reinforced polymer (CFRP), Al/SiC particulates, and Glass fibre. Among various composites $\mathrm{Al}-\mathrm{SiC}$ MMCs was investigated by number of researchers as it find many engineering application. The turning process employ carbide and PCD tool due to hard nature of abrasive particles used. Number of the researcher modeled the turning process for prediction of surface roughness. The modeling of surface roughness, tool wear, and cutting force were main contribution in this area.

El-Gallab and Sklad (1998) have conducted number of machining experiments for selection of the optimum tool material, tool geometry and cutting parameters for the turning of $\mathrm{Al}-\mathrm{SiCp}$ (20\%) using coated carbide and polycrystalline diamond (PCD) tool and found that PCD has longer tool life. Number of researchers developed a model for prediction of surface roughness on $\mathrm{Al}-\mathrm{SiCp}$ MMC in the turning process. (Davim, 2003) has applied orthogonal array and analysis of variance (ANOVA) to investigate the cutting characteristics of MMC (A356/20/SiCp-T6) in turning using PCD cutting tool. He found that cutting velocity has the highest physical and statistical influence on the tool wear and cutting power required to perform the machining operation. Feed has highest physical and statistical influence in surface roughness. Muthukrishnan and Davim (2008) have 
conducted an experimental study on surface roughness in turning of Al-SiCp (20\%) MMC using PCD tool. They found that the feed rate is highly influencing parameter in ANOVA analysis. In neural network model the prediction found closer with experimental data and concluded that $\mathrm{NN}$ is better in terms of higher accuracy and less time is need for model development. Manna and Bhattacharya (2005) studied the influence of cutting speed on the forces developed during turning of Al-SiCp composites. The experimental results showed that 'feed force' and 'cutting force' decreased with an increase in the cutting speed. Davim (2008) developed multiple linear regression models for prediction of tool wear, power consumed and surface roughness obtained during turning of A356/20/SiCp-T6 metal matrix composite. The model prediction and the confirmation experiments show that the maximum error on tool wear prediction and it is $10 \%$. The maximum error in power consumed and surface roughness prediction found to be $3.2 \%$ and $10.3 \%$ respectively. (Kilickap et al, 2005) conducted an experimental study on homogenized $5 \%$ $\mathrm{SiC}_{\mathrm{p}}$ aluminum MMC material for the investigation of tool wear and surface roughness by turning process. They have used three influencing parameters viz. cutting speed, feed rate and depth of cut and found that tool wear is mainly affected by cutting speed and also prefer coated cutting tool than uncoated.

Apart from MMCs few researchers conducted experiments on machining Glass Fiber Reinforced Plastics (GFRPs) and PEEK composite materials. (Palanikumar et al., 2006) conducted machining experiments on GFRPs using PCD cutting tool. They attempted to obtain machining parameters for minimum surface roughness employing Taguchi method and RSM. They concluded that fiber orientation and machining time are more influencing parameter on machining GFRP composites. (Rajasekaran et al, 2011) conducted machining experiments on CFRP composites using cubic boron nitride (CBN) cutting tool. They applied fuzzy logic technique for modeling of surface roughness and found that feed is an influencing parameter. The predictive capability of FL found better. Antonio et al., (2008) used ANN modeling and GA optimization methodology for optimizing orthogonal turning process of PEEK composites to obtain optimum cutting conditions. Chandrasekaran et al., (2012) employed artificial neural network for developing surface roughness model for end milling of Al-SiCp MMC using carbide tool. They found that the model predicts with average prediction error $0.31 \%$ when compared with experimental data. The surface roughness is mainly affected by feed rate followed by spindle speed and depth of cut. They have also compared the performance of ANN model with RSM and found that ANN is better in prediction.

In this work an experimental investigation on turning Al-SiCp composite was performed to study the effect of three machining parameters viz. spindle speed, feed rate and depth of cut, on the surface roughness and metal removal rate. The analysis has done on responses to find out the relationship with input parameters viz., spindle speed, feed rate and depth of cut. The behavior of the input parameters on the surface roughness and material removal rate are plotted in the form of graph for better analysis. ANN model is established for obtaining the relationship between the response and the input cutting parameters. The network predicts surface roughness and metal removal rate for unseen data and found that the result/prediction is better. For desired surface finish of the component to be produced the optimum cutting parameters are obtained among different combination of cutting parameters are available. The paper is organized as follows. The review of previous literature is presented in Section 1. Section 2 demonstrates experimental study on surface roughness and metal removal rate and ANN model is presented in Section 3.

\section{Experimental work}

In this work, turning of Al-SiCp (5\%) MMC was performed using PCD tool. The machinability study and development of predictive modeling between the cutting parameters and output response were carried out. For the selection of cutting parameters the available literature reveals that spindle speed/cutting speed, feed rate and depth of cut were three primary machining parameters on which surface roughness and metal removal rate depends. In the present study, spindle speed, feed rate and depth of cut was considered as machining parameters. Three parameters each of three levels, varying one factor at a time, having $3^{3}=27$ full factorial design of experimentation based on Taguchi $\mathrm{L}_{27}$ orthogonal array of experiments were conducted. The factors i.e., spindle speed, feed, and depth of cut (axial) available/possible in the machine and considered for the study is shown in Table 1.

Table 1 Experimental parameters and level

\begin{tabular}{|l|l|l|l|l|}
\hline \multirow{2}{*}{ Factors } & \multirow{2}{*}{ Notation } & \multicolumn{2}{|l|}{ Levels } \\
\cline { 3 - 5 } & & 1 & 2 & 3 \\
\hline Speed (rpm) & $N$ & 500 & 775 & 1200 \\
\hline Feed rate & \multirow{2}{*}{$f$} & 0.11 & 0.22 & 0.44 \\
\hline (mm/rev) & & & & \\
\hline Depth of cut $(\mathrm{mm})$ & $d$ & 0.5 & 0.75 & 1 \\
\hline
\end{tabular}

The surface roughness and material removal rate were obtained for each experimental trial. The centre line average (CLA) value of surface roughness is considered as measure of job quality. The material removal rate as measure of productivity was evaluated. It is the total volume of metal removed in a unit time. It is given by the Eq. 1

$M R R=1000 v f d \quad \mathrm{~mm}^{3} / \mathrm{min}$

(or)

$M R R=\pi D N f d$

where $N$ is spindle speed (rpm), $v$ is cutting speed $(\mathrm{m} / \mathrm{min}), D$ is diameter of the workpiece $(\mathrm{mm}), f$ is feed rate $(\mathrm{mm} / \mathrm{rev})$ and $d$ is depth of cut $(\mathrm{mm})$.

The machining experiments were carried out on centre lathe of Kirloshkar make (Model: M1330). The Al/SiCp cylindrical workpiece of $60 \mathrm{~mm}$ diameter is held and 
rotated on 4 jaw chuck. The PCD tool is clamped on tool holder. Figure 1(a) shows the machining set up of the turning process. The machining was performed by selecting different combination of machining parameters. The machining length was $150 \mathrm{~mm}$. After each trial the surface roughness is measured length wise as well as in radial direction at three different place and average is taken. The CLA surface roughness $\left(R_{a}\right)$ values are measured using Pocket Surf (Mahr, GMBH) surface roughness measuring instrument. Its measuring range was $0.03-6.35 \mathrm{~mm}$. The surface roughness evaluation length in each case was taken as $2.4 \mathrm{~mm}$. This considering $20 \mu \mathrm{m}$ size of $\mathrm{SiC}$ particulate used in the work material. Figure 1(b) shows the set up for surface roughness measurement of machined component. The experimental outcome/responses of all 27 machining trials were presented in the Table 2 .
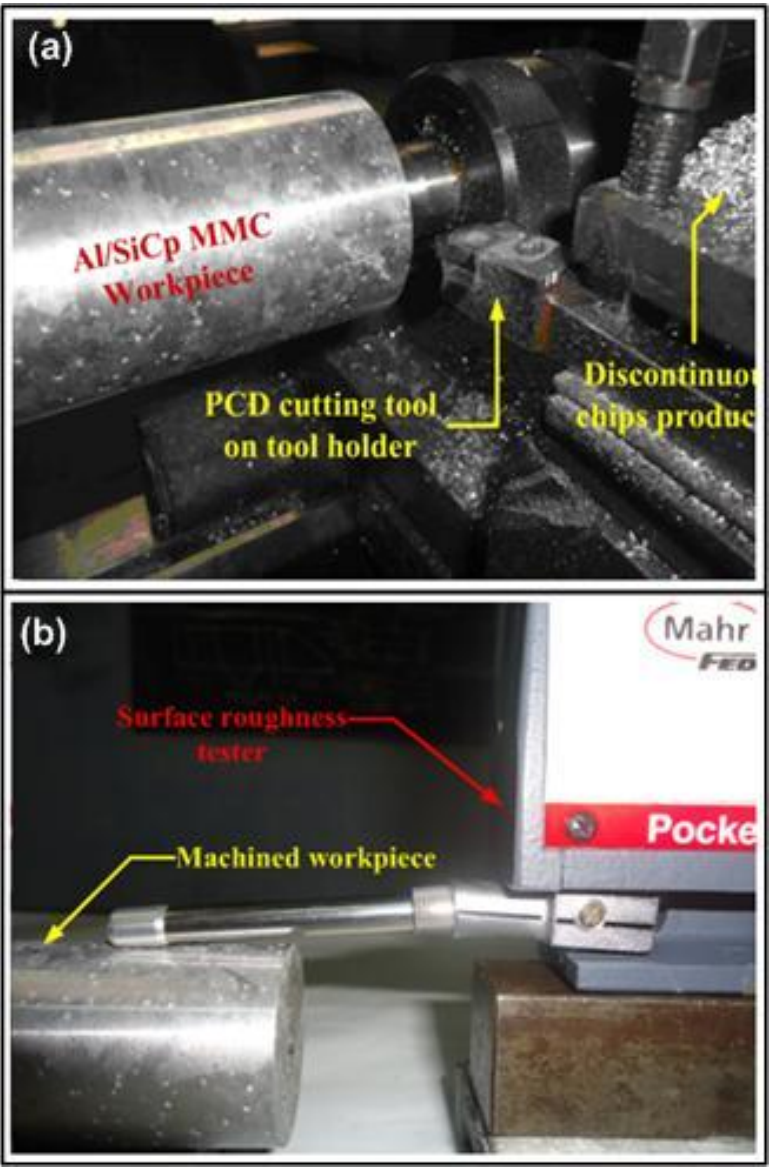

Fig. 1 (a) Machining set up and (b) Measurement of surface roughness

The observation of experimental data reveals that all three parameters influences surface roughness and material removal rate. The parameter influence on the response were studied and presented through graphical plot in the next sub section.

\subsection{Behavior of process parameter on the responses}

After experimentation the analysis have done on responses to find out the relationship with input parameters viz., spindle speed, feed rate and depth of cut. The behavior of the input parameters on the surface roughness and material removal rate are plotted in the form of graph for better analysis.

Table 2 Experimental results

\begin{tabular}{|c|c|c|}
\hline \multirow[t]{2}{*}{ Expt. trials } & \multicolumn{2}{|c|}{ Performance measures } \\
\hline & $R_{\mathrm{a}}(\mu \mathrm{m})$ & $\operatorname{MRR}\left(\mathrm{cm}^{3} / \mathrm{min}\right)$ \\
\hline 1 & 1.33 & 3.46 \\
\hline 2 & 1.30 & 5.19 \\
\hline 3 & 1.55 & 6.91 \\
\hline 4 & 2.44 & 6.91 \\
\hline 5 & 2.51 & 10.37 \\
\hline 6 & 2.81 & 13.83 \\
\hline 7 & 4.60 & 13.83 \\
\hline 8 & 5.43 & 20.74 \\
\hline 9 & 5.66 & 27.66 \\
\hline 10 & 1.28 & 5.36 \\
\hline 11 & 1.29 & 8.04 \\
\hline 12 & 1.50 & 10.72 \\
\hline 13 & 2.29 & 10.72 \\
\hline 14 & 2.45 & 16.08 \\
\hline 15 & 2.74 & 21.43 \\
\hline 16 & 4.85 & 21.43 \\
\hline 17 & 5.12 & 32.15 \\
\hline 18 & 5.29 & 42.87 \\
\hline 19 & 1.25 & 8.30 \\
\hline 20 & 1.27 & 12.45 \\
\hline 21 & 1.50 & 16.59 \\
\hline 22 & 2.38 & 16.59 \\
\hline 23 & 2.29 & 24.89 \\
\hline 24 & 2.64 & 33.19 \\
\hline 25 & 3.81 & 33.19 \\
\hline 26 & 4.78 & 49.78 \\
\hline 27 & 5.19 & 66.38 \\
\hline
\end{tabular}

\subsubsection{Variation of surface roughness}

The variation of surface roughness with different process parameter is graphically plotted in Figure $2(\mathrm{a}-\mathrm{c})$ Figure 2 (a) shows the variation of $R_{\mathrm{a}}$ with $N$. It shows from the graph that $R_{\mathrm{a}}$ does not have any significant effect on the surface roughness at lower feed rate. However the surface quality improves at higher spindle speed with increased feed rate. The high power consumption at increased spindle speed overcomes the feed effect causes improved surface roughness.

The variation of $R_{\mathrm{a}}$ with $f$ is depicted graphically in Figure 2 (b). It can be inferred from the graph that $R_{\mathrm{a}}$ increases with increase in $f$. The rapid movement of cutting tool as feed increases detoriates the surface roughness. The lower value of $f$ and higher value of $N$ shows improved surface roughness.

The influence of $d$ on surface roughness is shown graphically in Figure 2(c). The influence of $d$ on $R_{a}$ is not much significant. However, it can be seen from the graph that $R_{\mathrm{a}}$ increases slightly with increases in $d$. This is due to higher cutting load. The combination of lower value of $f$ and $d$ with high spindle speed obtain minimum surface roughness.

3 | International Conference on Advances in Mechanical Sciences 2014 

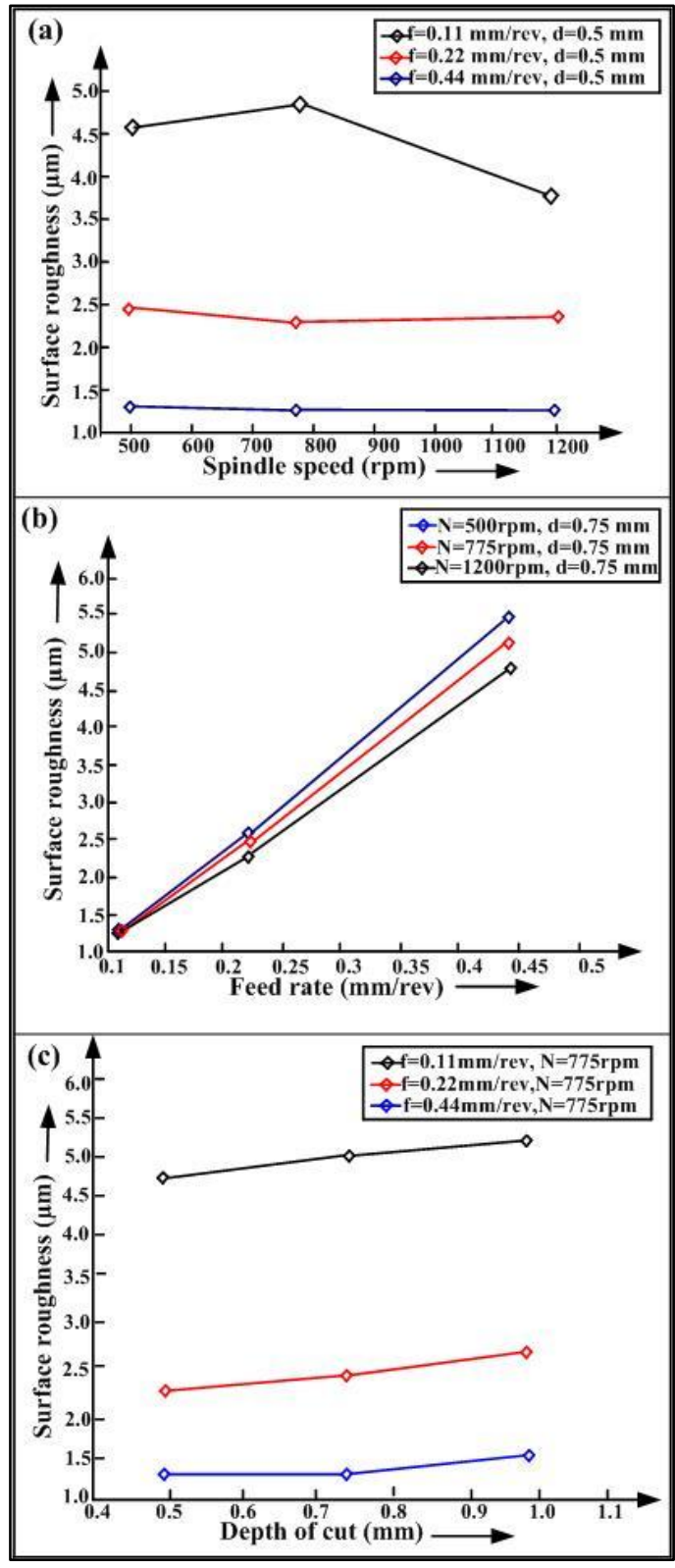

Fig. 2 Effect of (a) Spindle speed (b) Feed rate and (c) Depth of cut on the surface roughness

\subsubsection{Variation of material removal rate}

The variation of material removal rate with respect to different cutting condition is shown in Figure $3(\mathrm{a}-\mathrm{c})$. The variation of material removal rate with $N$ is shown in Figure 3 (a). The graph reveals that $M R R$ is directly proportion with $N$ when $f$ and $d$ are kept constant. The variation of material removal rate with $f$ is depicted graphically in Figure 3 (b). The feed also influence similar to $N$. To increase the MRR selection of feed should be higher. Since higher feed increases $R_{\mathrm{a}}$ proper selection of $\mathrm{f}$ for improved quality and increased MRR to be decided.

The effect of $d$ on material removal rate is shown graphically in Figure 3 (c). It can be seen from the graph that $M R R$ increases with increases in $d$. At lower value of $d, N$ and $f$ the material removal rate is lower as the value of parameters increase the $M R R$ starts increasing.

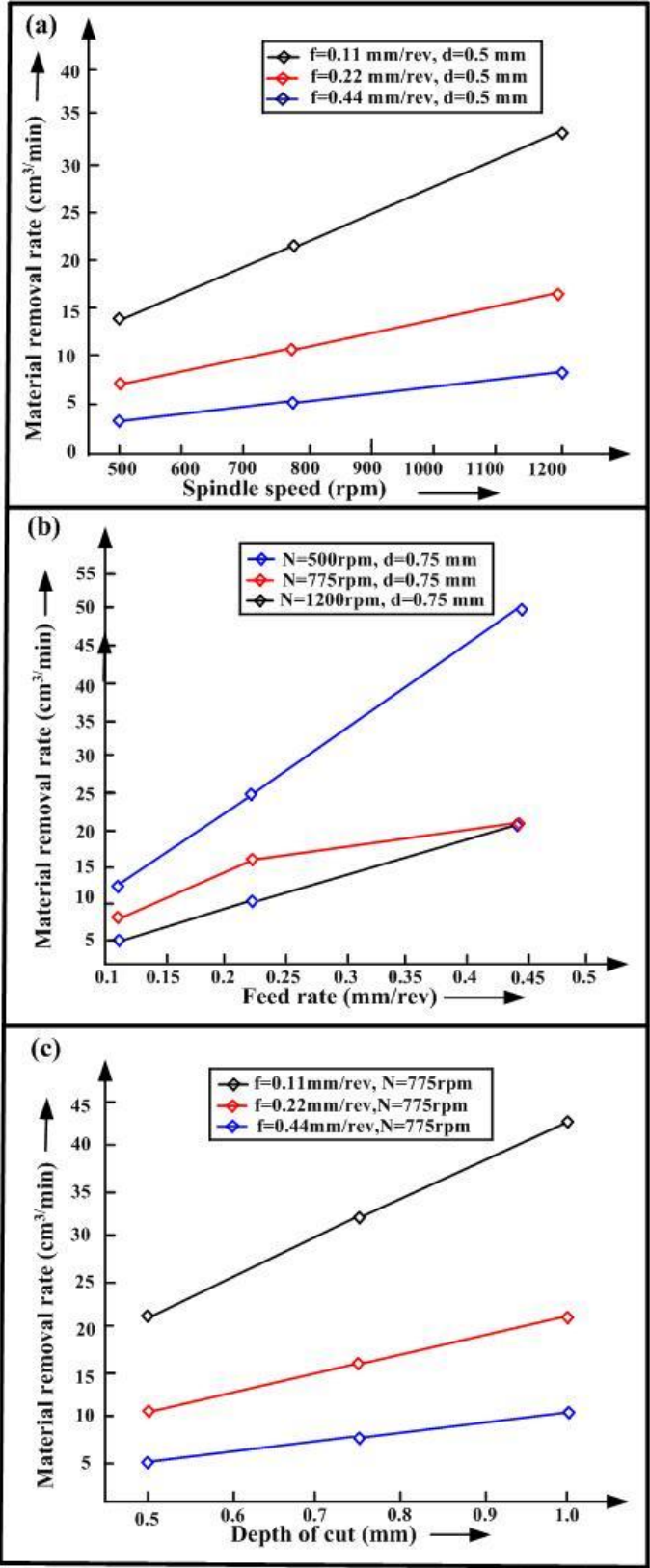

Fig. 3 Effect of (a) Speed, (b) Feed rate and (c) Depth of cut on material removal rate

\section{Development of multi response predictive model using ANN}

The artificial neural network (ANN) is a data processing system consisting of a large number of simple and highly interconnected processing elements resembling biological neural system. It can be effectively used to determine the input-output relationship of a complex process and is considered as a tool in non-linear statistical data modeling (Dixit and Dixit, 2008). The concept of artificial neuron network (ANN) was first proposed and introduced by McCulloch and Pitts in the year 1943 and was referred as the threshold logic gate (TLU) or linear threshold gate (LTU). It performed weighted sum operation followed by threshold operation. The neuron will fire and emit instantaneous 1 for the cases the threshold exceeded the 
weighted sum, otherwise emits -1 . Figure 4 shows graphical of representation of an artificial neuron. The first perceptron - the learning machine was introduced by Rosenblatt in the 1958. The neuron was trained to adjust its weight and bias by supplying set of input data and the corresponding targets

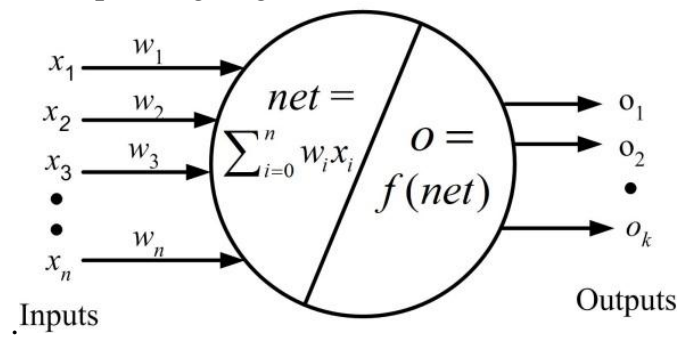

Fig. 4 An artificial neuron in ANN Model

Neural networks of human brain system perform three functions viz., learn, keep remember (i.e., store) and apply that knowledge that are gained from day to day activities. Similarly an artificial neural network (ANN) is developed which can be used for modeling complex manufacturing process with an ability to learn the process through data set obtained by conducting machining experiments. Researchers found easy application of the technique for process modeling with great success compared to other traditional modeling techniques (Kaw, 2008). It is used to build prediction models in machining optimization problems viz., surface roughness prediction, tool life prediction, cutting force prediction, etc.

Typical ANN model consists of an input layer having number of neurons for presenting data to the network, one or more hidden layer (number of neurons are decided upon network optimization) and an output layer present model response. To develop a predictive model the network is initially trained with set of experimental data sets. During the training process the network adjusts its interconnected weights between neurons of adjacent layers. The error between predicted value of the model and experimental value (i.e., desired outputs) is minimized. The optimum network is arrived upon minimum error by randomly varying weights. The two types of $\mathrm{NN}$ models are multi layer perceptron (MLP) feed forward network and radial basis function (RBF) network while MLP with single hidden layer network is commonly used for model development.

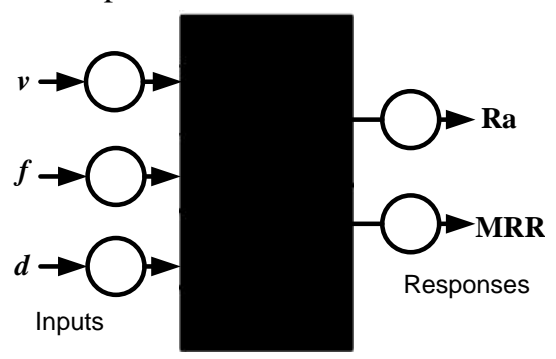

Fig. 5 ANN Model for prediction of $R_{\mathrm{a}}$ and $M R R$ in Al$\mathrm{SiCp} \mathrm{MMCs}$

Figure 5 shows a typical ANN model as black box used for prediction of surface roughness $\left(R_{\mathrm{a}}\right)$ and material removal rate $(M R R)$ of $\mathrm{Al}-\mathrm{SiCp}$ composites for turning process using PCD tool. The spindle speed $(N)$, feed rate $(f)$ and depth of cut $(d)$ are considered as input variables and thus input layer consists of 3 neurons and a two neurons at its output layer. ANN model is advantageous with other predictive model because of their reasonable accuracy and involves lesser computational time. Some drawbacks are it requires voluminous data set for network training and testing, the network predicts poor for extrapolated data set.

\subsection{ANN architecture}

The network with neurons (nodes) in each layers are interconnected with nodes of the subsequent and preceding layer with synaptic weights. Additionally a bias is added to each neurons of the hidden and output layer. The output of each neuron is obtained by summing up weighted inputs of neurons in preceding layer and its own bias. The output of each neuron in the hidden or output layer is computed by the Eq. 3 .

$$
\text { out }_{i}=f\left(\text { net }_{1}\right)=f\left(\sum_{w_{i j}} \text { out }_{j}+\theta_{1}\right) \text {, }
$$

where out ${ }_{i}$ is the output of the $i^{\text {th }}$ neuron in the layer under consideration; out $j_{j}$ is the output of the $j^{\text {th }}$ neuron in the preceding layer.

Figure 6 shows the architecture of two layered feed forward neural network system used in this work. The network is modeled with neural network tool box available in MATLAB 7.1 that working on back propagation learning algorithm. The algorithm use gradient decent technique and minimize mean square error (MSE) between actual network outputs with desired output pattern.

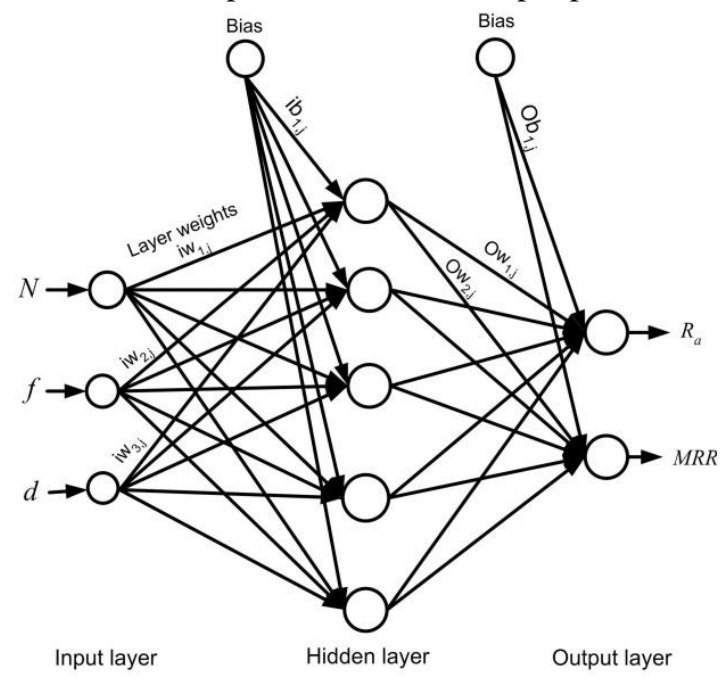

Fig. 6 ANN architecture

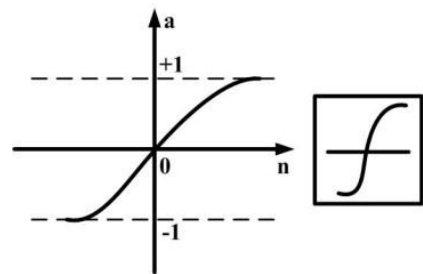

(a) 'Logsig' for hidden layer

5 | International Conference on Advances in Mechanical Sciences 2014 


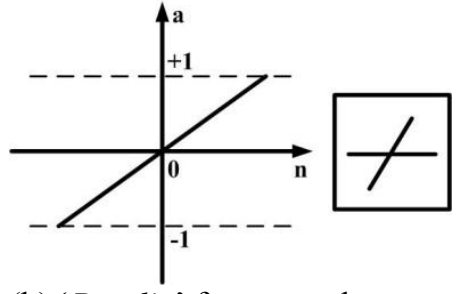

(b) 'Purelin' for output layer

Fig. 7 Transfer functions used

The number of neurons in the hidden layer plays a vital role in deciding the optimal architecture for the network. The network is optimized with varying number of neurons in the hidden layer and activation transfer function used so as to obtain minimum MSE. The network architecture with six hidden layer neurons with 'tansig' transfer function obtains least MSE of 0.00001 and is considered as optimum network. The output layer uses 'purelin' transfer function to evaluate the estimated outputs of surface roughness and material removal rate. The validation of the network is performed by predicting surface roughness and material removal rate for unseen data sets and ANN prediction is compared with experimental result. The neurons in the hidden layer should be increased gradually to avoid the problem of having too large neurons. It is also equally important to select the most suitable transfer function for hidden layer neurons. A transfer function calculates the output of a layer from its net input. The hyperbolic tangent sigmoid (tansig) transfer function and purelin transfer function work on the algorithm given in Eq. 4 and Eq. 5 respectively.

$$
\begin{aligned}
& a=\operatorname{tansig}(n)=\frac{2}{1+e^{-2 n}}-1 \\
& a=\operatorname{purelin}(n)=n,
\end{aligned}
$$

where $n$ is weighted input to neuron.

\subsection{Development of ANN modeling}

The model development consists of two stages i.e., training and testing. During network training the network architecture is trained so as obtain desired error goal. Finally the weights and bias of the optimum network is saved. While testing the network the trained net work is used for prediction of unseen interpolated data sets and the prediction is compared with experimental result. The procedure for neural network modeling is described as follows.

- Select input and response parameters of the model. This decides number of neurons required on input and output layer. Consider one hidden layer in between.

- Input error goal, maximum number of iterations (epochs), input and output pattern for network training. Set randomly the number of neurons in the hidden layer.

- Present training data pattern into the network.

- Randomly assign small values for weights and bias for the neurons connected in between the input, hidden and output layers. Evaluate the output values for all the neurons in hidden as well as output layers using Eq.5.4.

$$
\text { out }_{i}=\phi\left(\text { net }_{1}\right)=\phi\left(\sum_{w_{i j}} \text { out }_{j}+\theta_{1}\right)
$$

where out ${ }_{i}$ is the output of the $i^{\text {th }}$ neuron in the layer under consideration; out $j_{j}$ is the output of the $j^{\text {th }}$ neuron in the preceding layer. $\Phi$ is the tansig function can be expressed as:

$a=\operatorname{tansig}(n)=\frac{2}{1+e^{-2 n}}-1$,

where $\mathrm{n}$ is weighted input to neuron.

- Determine the output at the output layer and compare those with the desired output values. Evaluate the error $(E)$ of the output neurons and is given by,

$E=($ desired output - actual output $)$

Also evaluate the root mean square error value of the output neurons. It is given by Eq. (9).

$E_{p}=\frac{1}{2} \sum\left(t_{p j}-O_{p j}\right)^{2}$,

where $E_{\mathrm{p}}$ is the error for the $p^{\text {th }}$ presentation vector, $t_{p j}$ is the desired value for the $j^{\text {th }}$ output neuron and ${ }^{O_{p j}}$ is the desired output of the $j^{\text {th }}$ output neuron.

- Determine the error available at the neurons of the hidden layer and back propagate those errors to the weight values connected in between the neurons of the hidden layer and input layer. Similarly, back propagate the errors available at the output neurons to the weight values connected in between the neurons of the hidden layer and output layer using Eq. (10)

$\operatorname{Error} \delta_{p i}=\left(t_{p j}-O_{p j}\right) O_{p j}\left(1-O_{p j}\right)$

for output neurons

$\operatorname{Error}_{p i}=\left(t_{p j}-O_{p j}\right) O_{p j} \sum \delta_{p i} W_{k i}$

for hidden neurons.

- Weight adjustment is made as follows:

$\Delta W_{j i}(n=1)=\eta\left(\delta_{p i} O_{p i}\right)=\alpha \Delta W_{j i}(n)$,

where $\eta$ is the learning rate parameter and $\alpha$ is momentum factor.

- Repeat the iterative cycle until the desired condition is met. At the end of cycle determine the root mean square error value, mean percentage of error and worst percentage of error over the complete patterns.

- Stop the iteration and save the final weight and bias values associated the hidden layer neurons and also to the output layer neurons.

- Testing neural network model with the trained weight values, determine the output for the testing pattern and check whether the deviation from desired value is reasonably less or not. If no, try the back propagation 
with revised network by changing the number of neurons, altering learning rate parameters, altering momentum value and altering temperature value. The procedure is pictorially presented in the form of flow chart shown in Figure 8.

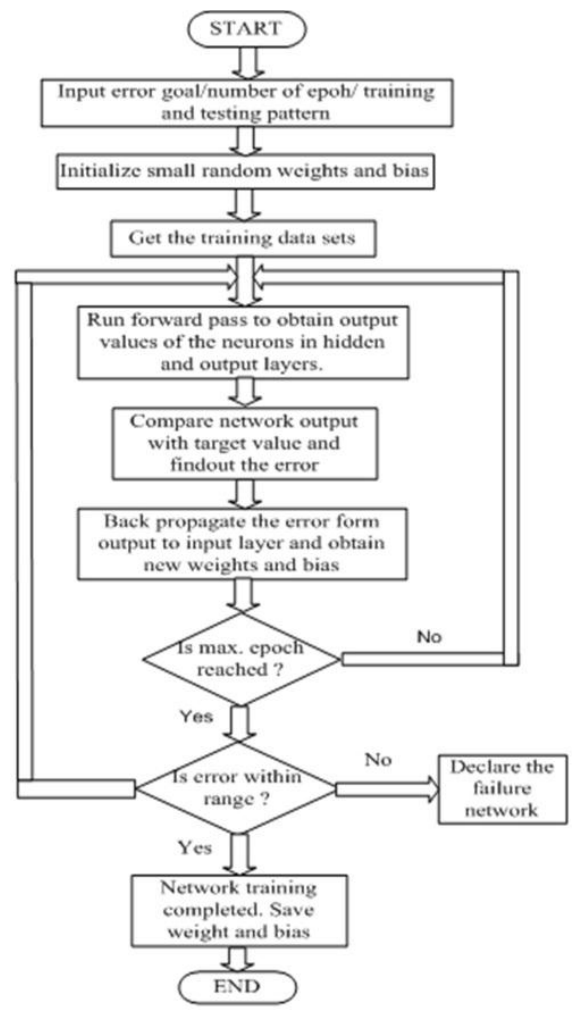

Fig. 8 Flow chart of ANN modeling

A properly trained network will give nearly equal training and testing error. Training error is the error in prediction for training data set which can be reduced by increasing the network parameters. Such a network exhibits poor generalization property and predicts poorly for new data set resulting in network over fitting. The network is tested with test data set and the error in prediction for the test data set is known as testing error. In the present work, network giving nearly equal amount of training and testing error is selected. The optimum number of of neurons and the corresponding transfer function is selected based on the effective error which is the difference between training and testing error. The training the network until one of the stopping criteria is met. The network configurations giving average percentage error in training and testing data set within $20 \%$ are considered. The $\mathrm{NN}$ architecture having $3-$ $6-2$ is found to be optimum average percentage error of $4.46 \%$ for surface roughness and $7.26 \%$ for material removal rate.

Amongst the various training functions available to train a multi layer network, the training function trainlm that works on the Levenberg-Marquardt back propagation algorithm is the most widely used. It is the fastest training function and the default for feed forward network in the MATLAB tool box as it is better on function fitting (nonlinear regression) problems. However, it becomes less efficient for larger network with thousands of weights as these require more memory and more computational time. The two methods available to improve generalization performance of neural network are early stopping and regularization. The optimal neural network architecture details including its weights and biases are given in the Table 3 .

Table 3 Weights and biases of the converged network

\begin{tabular}{|c|c|c|c|c|c|c|}
\hline \multicolumn{4}{|c|}{$\begin{array}{l}\text { Hidden Layer weights and bias } \\
\text { (HL) } \\
\text { Transfer function: 'tansig' } \\
\text { Number of neurons: six }\end{array}$} & \multicolumn{3}{|c|}{$\begin{array}{l}\text { Output Layer }(\mathrm{OL})=2 \\
\text { Transfer function : 'purelin' } \\
\text { Number of neurons: two }\end{array}$} \\
\hline \multicolumn{3}{|c|}{ Weights $\left({ }^{i w_{1 j}}\right)$} & \multirow{2}{*}{$\begin{array}{l}\text { Bias } \\
l b_{1, j}\end{array}$} & \multicolumn{2}{|c|}{ Weights } & \multirow{2}{*}{$\begin{array}{l}\text { Bias } \\
l b_{2, j}\end{array}$} \\
\hline$i w_{1 j}$ & $i w_{2 j}$ & $i w_{3 j}$ & & $l w_{2,1}$ & $l w_{2,1}$ & \\
\hline 1.16 & -5.98 & 2.63 & -8.22 & 0.37 & -0.27 & 0.83 \\
\hline-0.52 & -0.66 & -0.65 & 1.49 & 2.16 & -3.10 & 7.28 \\
\hline 3.85 & -6.65 & -1.61 & 4.04 & -3.97 & -5.30 & \\
\hline 0.41 & 3.38 & 0.48 & 1.37 & 1.43 & 0.50 & \\
\hline-3.21 & 5.17 & 1.43 & -3.62 & 2.56 & -5.45 & \\
\hline-7.55 & 11.70 & 3.00 & -7.25 & -5.26 & -0.21 & \\
\hline
\end{tabular}

\subsection{Model verification}

The developed ANN model is validated by eight interpolated data sets to test the model performance. The validation data test is randomly selected and is used for input pattern and used to predict the value of $R_{\mathrm{a}}$ and $M R R$ The average percentage error (APE) is obtained using Eq. 13.

$A P E=\frac{1}{n} \sum_{i=1}^{n}\left[\frac{a b s\left(\text { Expt_value }_{i}-A N N \operatorname{Pr} e d_{i}\right)}{\text { Expt_value }_{i}} \times 100\right]$,

Where $n$ is the number of validation data. The model predicted $R_{a}$ and $M R R$ values were compared with experimental values and percentage error was calculated. The results are presented in Table 4 and 5.

Table 4 Validation result of $R_{\mathrm{a}}$ ANN model

\begin{tabular}{|c|c|c|c|c|}
\hline \multirow[b]{2}{*}{ S1. No } & \multicolumn{4}{|c|}{ Surface roughness, $R_{a}(\mu \mathrm{m})$} \\
\hline & Expt & $\begin{array}{l}\text { ANN } \\
\text { pred. }\end{array}$ & $\begin{array}{c}\% \text { tage } \\
\text { error }\end{array}$ & $\begin{array}{c}\text { Pred. } \\
\text { accuracy }\end{array}$ \\
\hline 1 & 5.43 & 5.95 & 8.73 & 91.27 \\
\hline 2 & 2.74 & 2.66 & 2.9 & 97.1 \\
\hline 3 & 5.29 & 5.03 & 4.9 & 95.1 \\
\hline 4 & 1.5 & 1.48 & 1.33 & 98.67 \\
\hline \multicolumn{3}{|c|}{ Average percentage error } & 4.46 & \\
\hline \multicolumn{4}{|c|}{ Model accuracy } & 95.56 \\
\hline
\end{tabular}

Table 5 Validation result of $M R R$ ANN model

\begin{tabular}{|c|c|c|c|c|}
\hline \multirow[b]{2}{*}{ Sl. No } & \multicolumn{4}{|c|}{ Material removal rate, $M R R\left(\mathrm{~cm}^{3} / \mathrm{min}\right)$} \\
\hline & Expt. & $\begin{array}{l}\text { ANN } \\
\text { pred. }\end{array}$ & $\begin{array}{l}\% \text { age } \\
\text { error }\end{array}$ & $\begin{array}{c}\text { Pred. } \\
\text { accuracy }\end{array}$ \\
\hline 1 & 20.74 & 19.17 & 7.56 & 92.44 \\
\hline 2 & 21.43 & 22.74 & 5.76 & 94.24 \\
\hline 3 & 42.87 & 37.87 & 11.6 & 88.37 \\
\hline 4 & 16.59 & 17.3 & 4.1 & 95.9 \\
\hline \multicolumn{3}{|c|}{ Average percentage error } & 7.26 & \\
\hline \multicolumn{4}{|c|}{ Model accuracy } & 92.74 \\
\hline
\end{tabular}

7 | International Conference on Advances in Mechanical Sciences 2014 
The ANN model predicts surface roughness with APE of $4.46 \%$ with a maximum error of $8.73 \%$ while APE for material removal rate prediction is found as $7.26 \%$ with the maximum of $11.63 \%$ for one data set only. Model accuracy (MA) was computed as the average of individual accuracy on confirmation data set (Abdul et al., 2010). It is expressed by Eq. 14. The model accuracy of the developed model based on its predictive capability was found to be $95.26 \%$.

$M A=\frac{1}{n} \sum_{i=1}^{n}\left(1-\frac{\left|t_{i}-y_{i}\right|}{t_{i}}\right) \times 100$

where $t_{\mathrm{i}}$ is the target value and $y_{\mathrm{i}}$ is the predicted value of dataset. The graphical representation of the NN prediction for validation data set is depicted in Figure 9 (a-b).

Thus it can be concluded that the surface roughness and material removal rate value predicted from neural network model exhibit close correlation with the experimental result as confirmed by the validation test. The prediction accuracy of the $R_{a}$ found slightly higher than $M R R$.

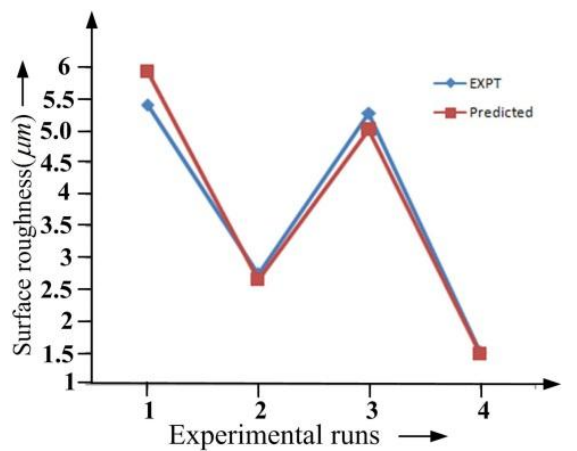

(a) For $\mathrm{Ra}$ (Max. error $8.73 \%$ )

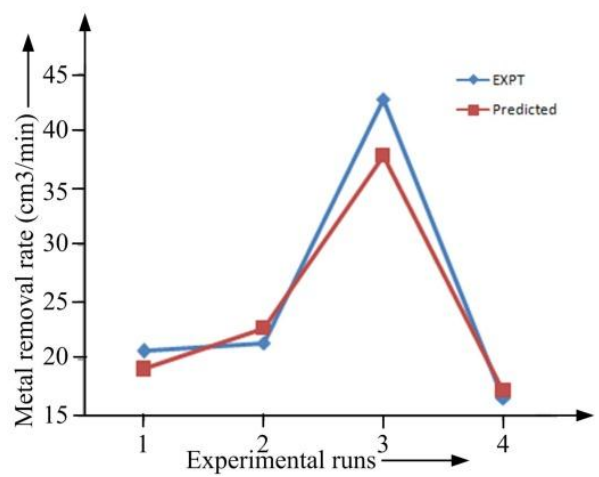

(b) For MRR (Max. error $11.63 \%$ )

Fig. 9 Experimental result Vs ANN prediction

\section{Conclusion}

In this work an experimental study and development of ANN predictive modeling in machining Al-SiCp MMC using PCD tool was carried out. The experimental study illustrates the behavior MMC material in turning process. The independent parameters such as spindle speed, feed and depth of cut were used to investigate two machining responses: (i) surface roughness as one of the important product quality of the manufactured component and (ii) material removal rate as productivity of the manufacturing process. Feed rate is highly influences the surface roughness followed by speed. The experimental result shows the increased feed detoriates surface roughness while increased speed improves it. Material removal rate increases with increase in feed followed by depth of cut and speed. The minimum surface roughness obtained as $1.25 \mu \mathrm{m}$ corresponding to the cutting conditions as $(N=1200 \mathrm{rpm}, f=0.11 \mathrm{~mm} / \mathrm{rev}$ and $d=0.5 \mathrm{~mm})$. The soft computing based ANN approach is used to develop multi response predictive model. The performance of the developed model is found encouraging with the average percentage of error as $4.46 \%$ for surface roughness and $7.26 \%$ for material removal rate. Realizing the lack of generality of the empirical model development ANN is found efficient and can be implemented for online application as intelligent tool.

\section{References}

J.E.Allison, G.S.Gole, (1993), Metal-matrix composites in the automotive industry opportunities and challenges, Journal of Minerals, Metallurgy and Materials, vol. 45, pp. 19-24.

M.ElGallab,M.Sklad,(1998),MachiningofAl/SiCparticulate metal matrix composites. Part 1. Tool performance, Journal of Material Processing Technology, vol 83, pp. 151-158.

J.P.Davim, (2003), Design of optimization of cutting parameters for turning of metal matrix composites based on the orthogonal arrays, Journal of Materials Processing Technology, vol. 132, pp. 340-344.

N.Muthukrishnan, J.P.Davim, (2009), Optimization of machining parameters of $\mathrm{Al} / \mathrm{SiC}-\mathrm{MMC}$ with ANOVA and ANN analysis, Journal of Materials Processing Technology, vol.209, pp. 225-232.

A.Manna, B.Bhattacharyya, (2005), Influence of machining parameters on the machinability of particulate reinforced $\mathrm{Al} / \mathrm{SiC}$ MMC, International Journal of Advance Manufacturing Technology, vol. 25, pp. 850-856.

E.Kilickap, O.C. Akir, A. Inan, (2005), Investigation of tool wear and surface roughness in turning of metal matrix composites, Journal of Materials Processing Technology, vol.164, pp. 862867.

K. Palanikumar, K. Karthikeyan, (2006),Optimal machining conditions for turning of particulate metal matrix composites using Taguchi and response surface methodologies, Machining Science Technology, vol. 10, pp. 417-433

T. Rajasekaran, K. Palanikumar, B.K. Vinayagam, (2011), Application of fuzzy logic for modeling surface roughness in turning CFRP composites using CBN tool, Production Engineering Res. Development, vol. 5, pp. 191-199.

C.O.C. Antonio, J.P. Davim, L. Vitor, (2008), Artificial neural network based on genetic learning for machining of polyetheretherketone composite materials, International Journal Advance Manufacturing Technology, vol. 39, pp. 1101-1110.

M.Chandrasekaran,D.Devarasiddappa,(2012),Development of Predictive Model for Surface Roughness in ends Milling of AlSiCp Metal Matrix Composites using Fuzzy Logic, World Academy of Science, Engineering and Technology, vol 68, pp. 1271-1276.

Dixit, P.M., and U.S. Dixit, (2008) Modeling of metal forming and machining processes by finite element and soft computing methods, Springer-Verlag, London.

K.A. Kaw, (2008) Mechanics of composite materials, Taylor \& Francis Group, Boca Rotan.

Abdul, S. M. J. Hashim, S. Z. M. Rahman, N.A (2010). Fuzzy logic based for predicting roughness performance of TiAIN coating, Proc.10th International Conference on Intelligent Systems Design and Applications, Malaysia, pp. 91-96. 